\title{
Why should localized gastric adenocarcinoma patients fare poorly after adjunctive therapy compared to surgery alone?
}

\author{
Jane E. Rogers ${ }^{1}$, Allison Trail ${ }^{2}$, Jaffer A. Ajani $^{2}$ \\ ${ }^{1}$ Pharmacy Clinical Programs, U.T. M.D. Anderson Cancer Center, Houston, TX, USA; ${ }^{2}$ Department of Gastrointestinal Medical Oncology, U.T. \\ U.T. M.D. Anderson Cancer Center, Houston, TX, USA \\ Correspondence to: Jaffer Ajani, M.D., U.T. M.D. Anderson Cancer Center, 1515 Holcombe Blvd, Houston TX 77030, USA. \\ Email: jajani@mdanderson.org.
}

Submitted Aug 13, 2020. Accepted for publication Oct 23, 2020.

doi: $10.21037 /$ cco-20-199

View this article at: http://dx.doi.org/10.21037/cco-20-199

Hypermutated phenotype that is particularly caused by microsatellite instability (MSI) has been in focus and concepts surrounding it are changing rapidly. Not only many of these tumor respond dramatically to checkpoint inhibition, some patients with metastatic cancer are practically cured. In this regard, we found the report by Pietrantonio et al. is of considerable interest (1). Their painstaking work to collect individual patient data and consuming analyses are commendable. Their results are unexpected and inconsistent with those noted for colon cancer. Additionally, these results are contradictory with two gastric adenocarcinoma (GAC) cohorts included in their meta-analysis and also published separately $(2,3)$. Restrategizing the management of MSI localized GAC is worthy of discussion. However, we raise several issues with their analysis and make suggestions.

As acknowledged by the authors, the entire analysis is based on only 121 total MSI GAC patients derived from four trials. In each cohort, an entirely unique adjunct strategy was used with two trials lacking a surgery alone control (1). The method for MSI assessment was not uniform and not known for the ITACA-S trial. Various statistical methods were used to accommodate a number of shortcomings in patient populations, as shown in their table 2, disease-free survival (DFS) and overall survival (OS) in the category for MSI tumor patients have starkly wide $95 \%$ confidence intervals compared to those in the microsatellite-stable (MSS) categories, making their observation less reliable and the conclusions weaker. The authors also did not explain why adjunctive systemic therapy would produce shorter DFS or OS for MSI GAC patients treated with adjunctive therapy. The MSI analysis of the CLASSIC trial (same 592 patients included in the Pietrantonio et al. report) showed no reduction in OS or DFS for MSI patients (3) and that of the ARTIST trial (almost the same patients included in the Pietrantonio et al. report) showed that MSI tumor patients actually fared better (the ARTIST trial lacked surgery alone control) (2). The Pietrantonio et al. report also uses the term predictive loosely to connote DFS/OS and many areas are confusingly presented (including, the * in table 2 is not explained, MSIlow can only be designated when polymerase chain reaction (PCR) is used to assess the MSI status) (1).

It is clear that MSI tumor patients (tumor type agnostically) have better prognosis when localized and in the advanced setting and have dramatic benefits from programmed-death-1 (PD-1) inhibition (4). Meaning, MSI tumors (despite their inherent heterogeneity) have rather uniform biologic/clinical characteristics. In this respect, we would like to think that if GAC patients fared poorly with adjunctive therapy, colon cancer patients should have experienced a disadvantage but that is not the case. The lack of benefit from certain adjunctive therapy in MSI tumor patients is a more consistent theme.

Finally, it is well known that the loss of function of tumor suppressor gene (TSG) (mutation/deletion, epigenomic

^ ORCID: 0000-0002-7275-7008 
silencing, or facilitation of TSG protein degradation) can result in upregulation of corresponding oncogenes (examples are TP53, CDH1, and ARID1A) (5). Work on mismatch repair TSGs is quite limited and needs to be expanded. Upregulation of oncogenes (when corresponding TSG protein function is lost) would make the cancer phenotypically aggressive and resistant to therapy (6). However, it is not likely to result in worse outcomes when cytotoxic agents are applied. Thus, we wonder if the conclusions of the current report should be moderated due to many limitations (small number of MSI tumor patients with wide confidence intervals for DFS and OS making the results/conclusions less robust, cohort heterogeneity, non-uniform therapeutic strategies, contradictory results published from two cohorts (one showing no detrimental effect on OS and another showing an advantage for MSI GAC patients), no validation of lack of benefit borne out in colon cancer studies which are cleaner and have large number of patients (7). We would also not agree that the localized MSI GAC patients should be stratified in empiric trials. We strongly suggest that these patients should not be included in generic trials but are in a need of unique strategies appropriate for their molecular/clinical behavior and some of these efforts are already underway (NCT04082572; NCT03832569). Such strategies will also afford a unique opportunity to study why nearly half of the MSI tumor patients do not benefit from PD-1 inhibition (is it the degree of MSI, neo-epitope load, neoantigen diversity/immunogenicity, T-cell diversity, quality of frame-shift-derived peptides, major histocompatibility complex-conformity/human leukocyte antigen diversity?). These MSI tumors are attractive targets for vaccines, cell therapies, simultaneous oncogene targeting therapies, and next generation of checkpoint inhibitors.

Finally, results of three new trials are worthy of mentioning. Chalabi et al. (8) reported a very novel strategy for MSI localized colon cancer patients who received preoperative nivolumab and ipilimumab and at surgery, many patients had complete pathologic response making this a viable future strategy. Ludford et al. reported another novel strategy in which patients with documented metastatic MSI colon cancer patients first received checkpoint inhibition (nivolumab) but later were taken to surgery to find complete pathologic response even though prior to surgery the CT scans were grossly abnormal in some patients (9). Finally on June 29, 2020, US Food and Drug Administration (www.fda.gov/) approved pembrolizumab as first-line immunotherapy for patients with MSI metastatic colorectal cancer.

\section{Acknowledgments}

Funding: None.

\section{Footnote}

Provenance and Peer Review: This article was a standard submission to the journal. The article was sent for external peer review.

Conflicts of Interest: All authors have completed the ICMJE uniform disclosure form (available at http://dx.doi. org/10.21037/cco-20-199). The authors have no conflicts of interest to declare.

Ethical Statement: The authors are accountable for all aspects of the work in ensuring that questions related to the accuracy or integrity of any part of the work are appropriately investigated and resolved.

Open Access Statement: This is an Open Access article distributed in accordance with the Creative Commons Attribution-NonCommercial-NoDerivs 4.0 International License (CC BY-NC-ND 4.0), which permits the noncommercial replication and distribution of the article with the strict proviso that no changes or edits are made and the original work is properly cited (including links to both the formal publication through the relevant DOI and the license). See: https://creativecommons.org/licenses/by-nc-nd/4.0/.

\section{References}

1. Pietrantonio F, Miceli R, Raimondi A, et al. Individual Patient Data Meta-Analysis of the Value of Microsatellite Instability As a Biomarker in Gastric Cancer. J Clin Oncol 2019;37:3392-400.

2. Miceli R, An J, Di Bartolomeo M, et al. Prognostic Impact of Microsatellite Instability in Asian Gastric Cancer Patients Enrolled in the ARTIST Trial. Oncology 2019;97:38-43.

3. Choi YY, Kim H, Shin SJ, et al. Microsatellite Instability and Programmed Cell Death-Ligand 1 Expression in Stage II/III Gastric Cancer: Post Hoc Analysis of the CLASSIC Randomized Controlled study. Ann Surg 2019;270:309-16. 
4. Le DT, Durham JN, Smith KN, et al. Mismatch repair deficiency predicts response of solid tumors to PD-1 blockade. Science 2017;357:409-13.

5. Morris LG, Chan TA. Therapeutic targeting of tumor suppressor genes. Cancer 2015;121:1357-68.

6. Wang R, Song S, Harada K, et al. Multiplex profiling of peritoneal metastases from gastric adenocarcinoma identified novel targets and molecular subtypes that predict treatment response. Gut 2020;69:18-31.

7. Romiti A, Rulli E, Pilozzi E, et al. Exploring the Prognostic Role of Microsatellite Instability in Patients

Cite this article as: Rogers JE, Trail A, Ajani JA. Why should localized gastric adenocarcinoma patients fare poorly after adjunctive therapy compared to surgery alone? Chin Clin Oncol 2021;10(3):32. doi: 10.21037/cco-20-199
With Stage II Colorectal Cancer: A Systematic Review and Meta-Analysis. Clin Colorectal Cancer 2017;16:e55-e59.

8. Chalabi M, Fanchi LF, Dijkstra KK, et al. Neoadjuvant immunotherapy leads to pathological responses in MMRproficient and MMR-deficient early-stage colon cancers. Nat Med 2020;26:566-76.

9. Ludford K, Cohen R, Foo WC, et al. Evaluation of complete pathological remission rates in surgically resected MSI-high metastatic colorectal cancers (mCRC). J Clin Oncol 2019;37:e15046. 